\title{
Educação Matemática na Educação Infantil: o estado de conhecimento no período de 2010-2019
}

\author{
Mathematical Education in Childhood Education: the state of \\ knowledge in the 2010-2019 period
}

\section{La Educación Matemática en la Educación Infantil: el estado del conocimiento en el período 2010-2019}

Thuanne Souza Jahnke ${ }^{1}$; João Carlos Pereira de Moraes $^{2}$

\section{RESUMO}

O presente trabalho visa analisar e caracterizar produções científicas que discutem Educação Matemática na Educação Infantil. Para tanto, realizou-se um levantamento com os descritores "Educação Matemática" AND "Educação Infantil" no Catálogo de Teses e Dissertações da Capes (CTDC), Biblioteca Digital Brasileira de Teses e Dissertações (BDTD), Portal de Periódicos da CAPES (PPC) e no Scientific Electronic Library Online - Scielo. Após primeira leitura, analisaram-se somente os estudos que possuíam crianças na condição de sujeito da investigação. Como resultado, notou-se que as pesquisas: (1) estão centradas na região Sudeste e tiveram um crescimento nos últimos cinco anos; (2) objetivam discutir o pensamento numérico, estocástico e geométrico na Educação Infantil; (3) o foco metodológico pauta-se na questão da ludicidade, principalmente sobre jogos e brincadeiras; (4) os registros imagéticos (filmagem, fotografia e desenho) são os instrumentos mais selecionados pelos pesquisadores da área; e (5) a perspectiva da psicologia construtivista tem sido a abordagem mais utilizada nos estudos. Por fim, consideramos a necessidade de expansão da pesquisa em Educação Matemática na Educação Infantil, bem como a ampliação de perspectivas socioculturais nas abordagens com crianças.

Palavras-chave: Educação Matemática; Educação Infantil; Estado do Conhecimento.

\begin{abstract}
This research aims to analyze scientific productions that discuss Mathematics Education in Early Childhood Education. To this end, a survey was carried out with the descriptors "Educação Matemática" AND "Educação Infantil" in the Capes Theses and Dissertations Catalog (CTDC), Brazilian Digital Library of Theses and Dissertations (BDTD), CAPES Journals Portal (PPC) and Scielo. After the first reading, only studies that had children as research subjects were analyzed. As a result, it was noted that the surveys: (1) are focused on the Southeast region and have grown over the past five years; (2) aim to discuss numerical, stochastic and geometric thinking in Early Childhood Education; (3) the methodological focus is based on the question of playfulness, mainly on games and games; (4) imagery records are among the instruments selected by researchers in the field; and (5) the perspective of psychology has been the most used approach in studies. Finally, we consider the need to expand research in Mathematics Education in Early Childhood Education, as well as the expansion of socio-cultural perspectives in approaches to children.
\end{abstract}

Keywords: Mathematical Education; Child education; State of Knowledge.

\footnotetext{
${ }^{1}$ Licenciada em Letras e Especialista em Alfabetização e Letramento. Mestranda em Educação do Programa de Pós-Graduação em Educação (PPGEdu) da Universidade Federal do Pampa (UNIPAMPA), Jaguarão/RS - Brasil. E-mail: thuannejahnke.aluno@unipampa.edu.br

2 Doutor em Educação, Mestre em Educação Científica e Tecnológica e Licenciado em Matemática e Pedagogia. Professor da Universidade Federal do Pampa (UNIPAMPA), Jaguarão/RS - Brasil. E-mail: joaomoraes@unipampa.edu.br.
} 


\section{RESUMEN}

Esta investigación tiene como objetivo analizar las producciones cientificas que discuten la Educación Matemática en la Educación Infantil. Para ello, se realizó una encuesta con los descriptores "Educação Matemática" $Y$ "Educação Infantil" en el Catálogo de Tesis y Disertaciones de Capes (CTDC), Biblioteca Digital Brasileña de Tesis y Disertaciones (BDTD), Portal de Revistas CAPES (PPC) y Scielo. Después de la primera lectura, solo se analizaron los estudios que tenían niños como sujetos de investigación. Como resultado, se observó que las encuestas: (1) están enfocadas en la región sureste y han crecido en los últimos cinco años; (2) tratar de discutir el pensamiento numérico, estocástico y geométrico en la Educación Infantil; (3) el enfoque metodológico se basa en la cuestión de la alegría, principalmente en los juegos y juegos; (4) los registros de imágenes se encuentran entre los instrumentos seleccionados por los investigadores en el campo; y (5) la perspectiva de la psicología ha sido el enfoque más utilizado en los estudios. Finalmente, consideramos la necesidad de ampliar la investigación en Educación Matemática en Educación Infantil, así como la ampliación de las perspectivas socioculturales en los enfoques a la infancia.

Palabras clave: Educación Matemática; Educación Infantil; Estado del conocimiento.

\section{INTRODUÇÃO}

A análise do campo investigativo faz-se necessária para a realização do balanço sobre o que está sendo tratado em um determinado campo do conhecimento. As pesquisas que abordam o chamado "estado do conhecimento ${ }^{3 "}$ visam a contribuir para a formação do campo teórico de uma área do conhecimento, mapeando publicações, elencando lacunas e possíveis caminhos a serem seguidos (FERNANDES; MORAES; PEREIRA, 2020).

Ao referirmos sobre Educação Infantil e Educação Matemática na condição de campo de pesquisa, como apresenta Moraes (2020), o debate tem sido escasso e assistemático. O autor ressalta que, mesmo dentro do Grupo de Trabalho 01- Matemática na Educação Infantil e Anos Iniciais do Ensino Fundamental, da Sociedade Brasileira de Educação Matemática (SBEM), as intenções caminham para discutir o ensino e aprendizagem da Matemática associado mais aos Anos Iniciais do Ensino Fundamental do que à Educação Infantil, o que nos leva a problematizar esse espaço na tentativa de compreendê-lo.

Assim, consideramos esse estudo como um "estado do conhecimento", uma vez que ensejamos um mapeamento de pesquisas realizadas na área da Educação Matemática na Educação Infantil. Para tanto, selecionamos as seguintes bases de dados: Catálogo de Teses e Dissertações Capes (CTDC); Biblioteca Digital Brasileira de Teses e Dissertações (BDTD); Portal de Periódicos da Capes (PPC) e Scientific Electronic Library Online - Scielo, no período de 2010 a junho de 2020.

Deste modo, o objetivo desse estado do conhecimento é analisar produções científicas que discutem Educação Matemática na Educação Infantil. O texto está dividido da seguinte maneira: (a) Aspectos metodológicos da composição do estado do conhecimento; (b) Metodologia da revisão; (c) Resultado da pesquisa; (d) Metodologia de análise dos trabalhos; e (e) Análise dos dados.

\footnotetext{
${ }^{3}$ Optamos pelo termo "estado do conhecimento" em detrimento de "estado da arte", uma vez que estes não são sinônimos. O "estado do conhecimento" abarca um setor de publicações sobre o tema estudado e diferencia-se do "estado da arte" pela delimitação de locais de busca. Por sua vez, o "estado da arte" abrange a área do conhecimento em seus diferentes aspectos e produções, não se atendo apenas em teses e dissertações, por exemplo. Ou seja, como ressaltam Romanowski e Ens (2006), a semelhança entre ambos está em almejar mapeamento de uma área, mas seus afastamentos referem-se à abrangência das bases de dados que se fundamentam para tal.
} 


\section{ASPECTOS METODOLÓGICOS DA COMPOSIÇÃO DO ESTADO DO CONHECIMENTO}

Nesta seção, nós apresentamos a organização metodológica do estado do conhecimento, bem como a sua apropriação para o levantamento dos estudos que discutem Educação Infantil e Educação Matemática.

\subsection{Metodologia da revisão}

Para a produção do estado do conhecimento o associamos ao processo de revisão de literatura. Segundo Ramos e Faria (2012), a revisão de literatura objetiva a seleção de fontes, estabelecendo um distanciamento de crenças pessoais ou julgamentos subjetivos motivados por coordenadas geográficas ou pela esfera de alguma comunidade acadêmica.

Nesse sentido, estruturamos e apresentamos um protocolo de pesquisa, com o intuito de oferecer uma sequência organizada e padronizada para a investigação (Imagem 1). Nela constam: (1) objetivo; (2) equações da pesquisa; (3) âmbito da pesquisa; (4) critérios de inclusão; (5) critérios de exclusão; (6) critérios de validade metodológica; (7) resultados; e (8) tratamento de dados.

Imagem 1: Etapas do protocolo de Revisão Sistemática

\section{OBJETIVOS}

Definir a problemática

\section{EQUAÇÕES DA PESQUISA}

Expressões ou palavras combinadas com AND, OR, NOT

\section{ÂMBITO DA PESQUISA}

Definição de base de dados

\section{CRITÉRIOS DE INCLUSÃO}

Definem que o estudo é aceitável naquele contexto

\section{CRITÉRIOS DE EXCLUSÃO}

Excluem os estudos que não obedecem ao âmbito definido

CRITÉRIOS DE VALIDADE METODOLÓGICA

Asseguram a objetividade da pesquisa

\section{RESULTADOS}

Devem ser registrados todos os passos

\section{TRATAMENTO DOS DADOS}

Filtrar e analisar criticamente os resultados com apoio de questões norteadoras

Fonte: Adaptado pelos autores a partir de RAMOS e FARIA (2012) 
A revisão de literatura pode ser feita seguindo os passos elencados, mas também podem ser adaptada, com supressões ou acréscimos. Para a nossa perspectiva de estudo, encontramos nessa série de procedimentos uma harmoniosa e adequada sintonia no âmbito das pesquisas produzidas nas Ciências da Educação.

Todos os passos da revisão literária possuem objetivos bem definidos, porém o último passo, tratamento de dados, precisa ser destacado pela importância do processo: Filtrar e analisar criticamente os resultados com apoio de questões norteadoras. Após a seleção de textos através da revisão de literatura, questões permitirão evidenciar elementos que interessam aos pesquisadores do campo investigado.

\subsection{Procedimentos da pesquisa}

Neste momento, detalharemos todo o processo realizado (Quadro 1) no levantamento dos estudos relacionando Educação Infantil e Educação Matemática.

Quadro 1: Descrição das etapas no processo dessa revisão sistemática

\begin{tabular}{|c|c|}
\hline ETAPA & DESCRIÇÃo \\
\hline Objetivos & $\begin{array}{c}\text { Identificar dissertações, teses e artigos que relacionem } \\
\text { Educação Matemática e Educação Infantil. }\end{array}$ \\
\hline Equações da pesquisa & "Educação Matemática" AND "Educação Infantil" \\
\hline Âmbito da pesquisa & $\begin{array}{c}\text { 1- Catálogo de Teses e Dissertações da Capes (CTDC); } \\
\text { 2- Biblioteca Digital Brasileira de Teses e Dissertações (BDTD); } \\
\text { 3- Portal de Periódicos da CAPES (PPC); } \\
\text { 4- Scientific Electronic Library Online- Scielo. }\end{array}$ \\
\hline Critérios de inclusão & $\begin{array}{c}\text { 1- Trabalhos produzidos nos últimos dez anos (2010 a 2020); } \\
\text { 2- Trabalhos produzidos em programas de ensino e educação; } \\
\text { 3- Produções com crianças como sujeitos de pesquisa. }\end{array}$ \\
\hline Critérios de exclusão & $\begin{array}{c}\text { 1- Pesquisas que não estejam completas; } \\
\text { 2- Textos indisponíveis na internet. }\end{array}$ \\
\hline Critérios de validade \\
metodológica
\end{tabular}

Fonte: Os pesquisadores.

Os resultados obtidos e a sua metodologia de análise são apresentados na próxima seção.

\section{A ANÁLISE DOS TRABALHOS}

Após a aplicação do protocolo de pesquisa, no Quadro 2, a seguir, apresentamos os estudos levantados no âmbito da pesquisa: 
Quadro 2: Lista de trabalhos encontrados na Revisão Sistemática

\begin{tabular}{|c|c|c|c|c|}
\hline Termo* & Natureza & Título & Autor & Ano \\
\hline T1 & Dissertação & $\begin{array}{l}\text { Passeios aleatórios e o conceito de chance na } \\
\text { Educação Infantil: uma análise instrumental }\end{array}$ & SANTOS & 2017 \\
\hline T2 & Dissertação & $\begin{array}{l}\text { As brincadeiras e as noções espaciais na Educação } \\
\text { Infantil }\end{array}$ & REZENDE & 2016 \\
\hline T3 & Dissertação & $\begin{array}{l}\text { Esquemas utilizados por crianças da educação } \\
\text { infantil em situações envolvendo o campo } \\
\text { conceitual de chance }\end{array}$ & ALMEIDA & 2017 \\
\hline T4 & Dissertação & $\begin{array}{l}\text { A classificação em crianças de pré-escola: } \\
\text { contribuições do flex memo }\end{array}$ & ALMEIDA & 2017 \\
\hline T5 & Dissertação & $\begin{array}{l}\text { Representação semiótica: uma perspectiva para a } \\
\text { construção do conceito de número na Educação } \\
\text { Infantil }\end{array}$ & DINIS & 2018 \\
\hline T6 & Dissertação & $\begin{array}{c}\text { A compreensão das relações inversas da divisão } \\
\text { por crianças nos Anos Iniciais de escolarização: um } \\
\text { estudo acerca da não explicitação numérica em } \\
\text { problemas }\end{array}$ & OLIVEIRA & 2014 \\
\hline T7 & Dissertação & $\begin{array}{l}\text { Modelagem matemática na Educação Infantil: } \\
\text { contribuições para a formação da criança }\end{array}$ & BELO & 2016 \\
\hline T8 & Dissertação & $\begin{array}{l}\text { Matemática, Educação Infantil e jogos de } \\
\text { linguagem: um estudo etnomatemático }\end{array}$ & RODRIGUES & 2010 \\
\hline T9 & Artigo & $\begin{array}{l}\text { Tarefas matemáticas para o desenvolvimento da } \\
\text { percepção de espaço na Educação Infantil: } \\
\text { potencialidades e limites }\end{array}$ & $\begin{array}{l}\text { MOREIRA; } \\
\text { GUSMÃO; MOLL }\end{array}$ & 2018 \\
\hline T10 & Dissertação & $\begin{array}{l}\text { A aprendizagem do conceito de número de } \\
\text { crianças do Infantil V: interações com o flex memo }\end{array}$ & BELO & 2018 \\
\hline T11 & Tese & $\begin{array}{c}\text { Sentido espacial de crianças na Educação Infantil: } \\
\text { entre mapas, gestos e falas }\end{array}$ & ZOGAIB & 2019 \\
\hline T12 & Dissertação & $\begin{array}{l}\text { Desenvolvimento do pensamento geométrico na } \\
\text { Educação Infantil: teorias e práticas }\end{array}$ & SIQUEIRA & 2019 \\
\hline T13 & Dissertação & $\begin{array}{c}\text { A literatura infantil e a matemática: um estudo } \\
\text { com alunos de } 5 \text { e } 6 \text { anos de idade da Educação } \\
\text { Infantil }\end{array}$ & CARVALHO & 2010 \\
\hline T14 & Dissertação & $\begin{array}{c}\text { O uso de material manipulativo e a produção de } \\
\text { desenhos no desenvolvimento do raciocínio } \\
\text { combinatório na Educação Infantil }\end{array}$ & SILVA & 2019 \\
\hline T15 & Dissertação & $\begin{array}{l}\text { Jogos de reflexão pura como ferramenta lúdica } \\
\text { para a aprendizagem matemática }\end{array}$ & PIN & 2016 \\
\hline T16 & Tese & $\begin{array}{l}\text { Devir - criança da matemática: experiências } \\
\text { educativas infantis imagéticas }\end{array}$ & CHISTÉ & 2015 \\
\hline T17 & Artigo & $\begin{array}{l}\text { O ensino de matemática na Educação Infantil: uma } \\
\text { proposta de trabalho com jogos }\end{array}$ & $\begin{array}{l}\text { MORAES } \\
\text { et al. }\end{array}$ & 2017 \\
\hline T18 & Artigo & $\begin{array}{c}\text { É possível ensinar estocástica para crianças da } \\
\text { Educação Infantil? Uma análise à luz da teoria de } \\
\text { Bruner }\end{array}$ & DIAS et al. & 2020 \\
\hline
\end{tabular}

* Para otimizar a análise dos dados, cada trabalho recebeu a nomenclatura T e um número, conforme a ordem levantada. Fonte: os pesquisadores.

A análise dos trabalhos encontrados na Revisão Sistemática foi dividida em dois momentos. O primeiro refere-se à análise de aspectos gerais dos trabalhos, em que nos detemos em informações como: a região, a natureza do trabalho e o ano. O segundo momento respaldou-se a partir de perguntas, estipuladas a priori e respondidas a partir do conteúdo do corpus de análise (Quadro 2): 
* Quais são os pensamentos matemáticos discutidos com crianças na Educação Infantil?

* Quais são as abordagens metodológicas de ensino desenvolvidas nos estudos?

* Quais são os instrumentos de pesquisas propostos nos trabalhos?

* Quais as principais referências teóricas que embasam os estudos?

Abaixo apresentamos tais momentos e as considerações a partir dos critérios utilizados no estudo.

\subsection{Aspectos gerais}

Referente ao âmbito geral, analisamos três aspectos: (1) região do Brasil; (2) natureza da pesquisa; e (3) ano. Neste primeiro momento, a intenção é compreender como os estudos de Educação Matemática e Educação Infantil se localizam no espaço e tempo brasileiro.

Abaixo, apresentamos a divisão dos trabalhos conforme as regiões do país.

Quadro 3: Região do Brasil dos trabalhos

\begin{tabular}{|c|c|c|}
\hline Região & Total de Trabalhos & Trabalhos \\
\hline Centro-Oeste & 2 & $\mathrm{~T} 2, \mathrm{~T} 15$ \\
\hline Nordeste & 5 & $\mathrm{~T} 1, \mathrm{~T} 3, \mathrm{~T} 6, \mathrm{~T} 10, \mathrm{~T} 14$ \\
\hline Norte & 1 & $\mathrm{~T} 4$ \\
\hline Sudeste & 7 & $\mathrm{~T} 5, \mathrm{~T} 11, \mathrm{~T} 12, \mathrm{~T} 13, \mathrm{~T} 16, \mathrm{~T} 18, \mathrm{~T} 9$ \\
\hline Sul & 3 & $\mathrm{~T} 7, \mathrm{~T} 8, \mathrm{~T} 17$ \\
\hline
\end{tabular}

Fonte: a pesquisa.

Nota-se a concentração de trabalhos na região Sudeste do Brasil (7), bem como o número baixo de estudos região Norte (1). Consideramos que esse diferencial remeta-se ao número significativo e superior de Programas de Pós-Graduação em Educação e Ensino na Região Sudeste, o que nos leva a entender a necessidade de expansão de mestrado e doutorado pelo país como um espaço de formação e intervenção social.

Referente à natureza das pesquisas, elencamos os dados no Quadro 4.

Quadro 4: Natureza dos trabalhos

\begin{tabular}{|c|c|c|}
\hline Natureza da pesquisa & Total de Trabalhos & Trabalhos \\
\hline Dissertação & 13 & $\begin{array}{c}\mathrm{T} 1, \mathrm{~T} 2, \mathrm{~T} 3, \mathrm{~T} 4, \mathrm{~T} 5, \mathrm{~T} 6, \mathrm{~T} 7, \mathrm{~T} 8, \\
\mathrm{~T} 10, \mathrm{~T} 12, \mathrm{~T} 13 \mathrm{~T} 14, \mathrm{~T} 15\end{array}$ \\
\hline Tese & 2 & $\mathrm{~T} 11, \mathrm{~T} 16$ \\
\hline Artigo & 3 & $\mathrm{~T} 9, \mathrm{~T} 17, \mathrm{~T} 18$ \\
\hline
\end{tabular}

Fonte: a pesquisa

A partir dos dados, percebe-se que há mais trabalhos de dissertação (13) e menos trabalhos de tese (2). Tal fato permite refletir sobre a não continuidade da temática Educação Infantil e Educação Matemática em pesquisas, uma vez que, teoricamente, estudos desenvolvidos em uma dissertação são elementos base para doutorandos comporem suas teses. Se extrapolarmos esses dados, podese pensar a necessidade de criação de rede de pesquisadores que invista, em longo prazo, no assunto.

No que concerne ao ano de publicação dos estudos, levantamos os seguintes dados: 
Quadro 5: Ano dos trabalhos

\begin{tabular}{|c|c|c|}
\hline Ano & Total de Trabalhos & Trabalhos \\
\hline 2010 & 2 & $\mathrm{~T} 8, \mathrm{~T} 13$ \\
\hline 2014 & 1 & $\mathrm{~T} 6$ \\
\hline 2015 & 1 & $\mathrm{~T} 16$ \\
\hline 2016 & 3 & $\mathrm{~T} 2, \mathrm{~T} 7, \mathrm{~T} 15$ \\
\hline 2017 & 4 & $\mathrm{~T} 1, \mathrm{~T} 3, \mathrm{~T} 4, \mathrm{~T} 17$ \\
\hline 2018 & 3 & $\mathrm{~T} 5, \mathrm{~T} 9, \mathrm{~T} 10$ \\
\hline 2019 & 3 & $\mathrm{~T} 11, \mathrm{~T} 12, \mathrm{~T} 14$ \\
\hline 2020 & 1 & $\mathrm{~T} 18$ \\
\hline
\end{tabular}

Fonte: a pesquisa.

Investigando as datas de publicação, nota-se que há mais trabalhos realizados no período de 2016 a 2019 (13) e menos trabalhos em 2014 e 2015 (2). Observa-se também que entre 2011 e 2013 não houveram trabalhos registrados. Mediante os números do último quadriênio, visualizamos uma tendência de crescimento da temática nos trabalhos, o que pode gerar impacto e novas teorizações para a área nos próximos anos.

Ao analisarmos os aspectos gerais, percebemos que, em relação ao local, a região Sudeste apresenta maior concentração de trabalhos e a região Norte a menor. Já sobre a natureza da pesquisa, constatamos que a maioria dos trabalhos são dissertações. $\mathrm{E}$, por fim, a respeito do ano das pesquisas que o último quadriênio (2016-2019) teve mais trabalhos produzidos em comparação ao período de 2010-2014, com somente 3 estudos.

\subsection{Análise a partir de perguntas a priori}

A seguir, desenvolvemos a análise com base nas perguntas, tendo como eixos quatro categorias: as ideias e noções matemáticas discutidas com crianças na Educação Infantil; as abordagens metodológicas de ensino desenvolvidas nos estudos; os instrumentos de pesquisa propostos nos trabalhos; e as principais referências teóricas que embasam os estudos.

\subsubsection{Quais são os pensamentos matemáticos discutidos com crianças na Educação Infantil?}

Foram identificados três pensamentos matemáticos trabalhados com as crianças: pensamento geométrico, pensamento estocástico e pensamento numérico. Ainda elencamos trabalhos que não especificaram as ideias e noções matemáticas.

Vale destacar a nossa priorização do termo noção em detrimento de conteúdo matemático. Assim como Moraes (2020), consideramos que o uso do termo noção permite um espaço mais flexível e transversal em relação aos objetos de ensino propostos na Educação Infantil, afastando-se de certo enrijecimento histórico que a terminologia conteúdo possa oferecer para esta etapa da educação escolar.

No Quadro 6, apresentamos os pensamentos matemáticos contemplados nos trabalhos. 
Quadro 6: Pensamentos matemáticos discutidos nos trabalhos

\begin{tabular}{|c|c|c|}
\hline Noção & Total de Trabalhos & Trabalhos \\
\hline Pensamento Geométrico & 5 & $\mathrm{~T} 2, \mathrm{~T} 9, \mathrm{~T} 11, \mathrm{~T} 12, \mathrm{~T} 16$ \\
\hline Pensamento Estocástico & 4 & $\mathrm{~T} 1, \mathrm{~T} 3, \mathrm{~T} 14, \mathrm{~T} 18$ \\
\hline Pensamento Numérico & 5 & $\mathrm{~T} 4, \mathrm{~T} 5, \mathrm{~T} 6, \mathrm{~T} 10, \mathrm{~T} 13$ \\
\hline Não especificado & 4 & $\mathrm{~T} 7, \mathrm{~T} 8, \mathrm{~T} 15, \mathrm{~T} 17$ \\
\hline
\end{tabular}

Fonte: a pesquisa

No primeiro grupo, encontramos cinco trabalhos que desenvolvem o pensamento geométrico. T2 tem por objetivo descobrir quais noções espaciais as crianças de duas turmas de educação infantil mobilizam ao brincar, e como as manifestam. Em T9 o objetivo consiste em analisar as potencialidades e limites de tarefas matemáticas, elaboradas com base nos critérios de idoneidade didática, para favorecer o desenvolvimento da percepção de espaço na criança da Educação Infantil. T11 busca analisar evidências do sentido espacial da turma, que emergiram de suas interações, brincadeiras e tarefas no espaço escolar. T12 visou a analisar o trabalho da geometria na Educação Infantil, bem como elaborar a partir da teoria das habilidades geométricas de Hoffer um material didático que envolve atividades virtuais. Já T16 objetiva pensar nas imagens produzidas por crianças de uma instituição pública de educação infantil, envolvendo tempo e espaços.

Perante os objetivos citados, percebemos que a intenção pedagógica recai em noções, sentido e percepções do espaço. Em nossa perspectiva, esses elementos são trazidos à tona para promover uma concepção mais abrangente do pensamento geométrico, capaz de potencializar, além da matemática, compreensões geográficas, históricas e sociais do sujeito.

No segundo grupo, encontramos quatro trabalhos que desenvolvem o pensamento estocástico. T1 visa a analisar as relações que ocorrem quando crianças da Educação Infantil resolvem tarefas, envolvendo o conceito de chance, mediadas pela maquete tátil. Nesse mesmo sentido, T3 também objetiva analisar os esquemas utilizados por crianças da Educação Infantil em situações que envolvem o campo conceitual de chance no contexto da maquete tátil. T14 busca analisar a influência do uso de material de manipulação e da produção de desenhos na resolução de problemas combinatórios. Já T18 tem por objetivo analisar as contribuições de uma prática pedagógica, desenvolvida para ensinar estocástica a crianças da Educação Infantil, à luz da teoria de ensino de Bruner, com estatística e combinatória.

Com a descrição anterior, visualizamos que os estudos em pensamento estocástico fundamentam-se em questões de probabilidade e combinatória, como o conceito de chance. Por outro lado, notamos a ausência de estudos que focalizem as noções estatísticas, tais como coleta e organização de dados e a comunicação de inferências.

No terceiro grupo, encontramos cinco trabalhos que desenvolvem o pensamento numérico. T4 teve como objetivo analisar as relações estabelecidas pelas crianças usando o pensamento classificatório no jogo da memória com o Flex Memo. T5 tem por objetivo investigar o papel do registro de representação semiótica na construção do conceito de número por crianças da Educação Infantil. T6 visa investigar a compreensão da criança na resolução de problemas de divisão, acerca das relações inversas entre quociente e divisor quando o dividendo é mantido constante, sem a presença explícita do número expressando quantidades. T10 visa a analisar a contribuição da interação com os jogos de Memória, Segredo da Caixa e Batalha proporcionados pelo Flex Memo para 
a ampliação do conceito de número de crianças do infantil V. Em T13 busca-se mostrar como o educador pode trabalhar com a literatura e a matemática, criando momentos na prática de sala de aula que propiciam a compreensão e a familiarização com a linguagem matemática em crianças de 5 e 6 anos da Educação Infantil de uma escola municipal de São Paulo.

No quarto grupo, não especificado, encontram-se estudos que o foco está em situações metodológicas, não ocorrendo o debate a priori de pensamentos matemáticos. Assim, levantamos estudos com modelagem matemática (T7), Etnomatemática (T8) e jogos (T15, T17).

Nota-se que os estudos pautam-se principalmente em aspectos relativos ao processo de construção do conceito de número, bem como possibilidades teórico-metodológicas para tanto.

As ideias e noções matemáticas discutidas na Educação Infantil nos trabalhos analisados apresentam: no pensamento geométrico o foco em noções espaciais; no pensamento estocástico percebemos alguns conceitos estocásticos nas relações sobre estatística, probabilidade e combinatória; e no pensamento numérico observamos os objetivos voltados para o conceito de número.

Vale ressaltar que temas relativos à Grandeza e Medidas e Pensamento Algébrico não apareceram em nenhum dos estudos, essa questão permite-nos indicar a necessidade de debater tais temas na Educação Infantil.

\subsubsection{Quais são as abordagens metodológicas de ensino desenvolvidas nos estudos?}

Foram identificadas oito abordagens metodológicas de ensino nos estudos. Tais como: jogos, brincadeiras, resolução de problemas, modelagem matemática, mapas, material manipulável e desenho, produções imagéticas, e atividades virtuais. Também houve trabalhos que não especificaram a abordagem metodológica de ensino.

Quadro 7: Abordagens metodológicas de ensino desenvolvidas nos estudos

\begin{tabular}{|c|c|c|}
\hline Abordagem metodológica & Total de trabalhos & Trabalhos \\
\hline Jogos & 8 & $\mathrm{~T} 1, \mathrm{~T} 3, \mathrm{~T} 4, \mathrm{~T} 5, \mathrm{~T} 10, \mathrm{~T} 13, \mathrm{~T} 15, \mathrm{~T} 17$ \\
\hline Brincadeiras & 2 & $\mathrm{~T} 2, \mathrm{~T} 13^{4}$ \\
\hline Resolução de problemas & 1 & T6 \\
\hline Modelagem matemática & 1 & $\mathrm{T7}$ \\
\hline Mapas & 1 & T11 \\
\hline $\begin{array}{c}\text { Material manipulável e } \\
\text { desenho }\end{array}$ & 1 & T14 \\
\hline Produções imagéticas & 1 & T16 \\
\hline Atividades virtuais & 1 & T12 \\
\hline Não especificado & 3 & T8, T9, T18 \\
\hline
\end{tabular}

Fonte: a pesquisa

No primeiro grupo, encontramos oito trabalhos com abordagem metodológica de ensino desenvolvida a partir dos jogos. T1 utiliza maquete tátil com o jogo "Os passeios aleatórios do Jefferson três amigos" com crianças da Educação Infantil de 5 anos. T3 também utilizou maquete tátil composta de

\footnotetext{
${ }^{4}$ T13 está presente em dois momentos no quadro, uma vez que possui as duas abordagens - jogos e brincadeiras.
} 
peças e da sequência de ensino Passeios Aleatórios do Jefferson três amigos com crianças de 5 anos de idade. T4 usou o brinquedo Flex Memo para jogar memória com crianças em idade pré-escolar.

Ainda no primeiro grupo, T5 apresenta jogos ressignificados no Grupo de Estudo e Pesquisa em Aprendizagem e Educação Matemática (GEPAEM) com crianças de 5 anos. T10 utilizou jogos de memória Segredo da Caixa e Batalha proporcionados pelo Flex Memo com crianças do infantil V. T13 trabalha a linguagem matemática a partir de jogos que envolvam lateralidade e contagem com crianças de 5 a 6 anos de idade. Já T15 usou jogos de reflexão pura para estimular o pensamento e o raciocínio de crianças do $2^{a}$ período da Educação Infantil. Por fim, T17 utilizou o jogo Desafio dos ovos com crianças no período pré-escolar.

No segundo grupo, temos dois trabalhos que apresentam as brincadeiras como abordagem metodológica de ensino. T2 utilizou cinco brincadeiras relacionadas à exploração do espaço com crianças de 5 anos: cabra-cega, ajudando a chapeuzinho, vou de táxi, quatro cantos e caça ao tesouro. E T13 além dos jogos apresenta as brincadeiras como abordagem metodológica de ensino com crianças de 5 a 6 anos.

Tais trabalhos apoiam-se na perspectiva do lúdico como potencializador do trabalho pedagógico na Educação Infantil. O jogo e a brincadeira, como componente do lúdico, permitem às crianças elaborarem formas diversas de pensar o mundo, criando argumentos, estratégias e espaços para exercer a liderança (BROUGÈRE, 2002). Nesse sentido, no campo da Educação Matemática, propor práticas lúdicas pode ser um componente a ser aprofundado quando pensamos na Infância.

No grupo resolução de problemas, T6 utiliza problemas de divisão com quantidades explicitadas e com objetos de manipulação com crianças da Educação Infantil e dos dois primeiros anos do Ensino Fundamental. No grupo modelagem matemática, T7 usa diferentes atividades de modelagem matemática com crianças de 4 a 5 anos.

Nesses dois grupos citados, a situação problema torna-se elemento essencial. Ela é percebida como um aspecto propulsor e desencadeador da aprendizagem da criança. Tais práticas inserem espaços para a criança pensar a realidade, problematiza-la e organiza-la a partir do pensamento matemático, possibilitando sua intervenção.

No grupo mapas, T11 utilizou mapas, gestos e fala para trabalhar o sentido espacial com crianças de 4 a 6 anos de idade. No grupo material manipulável e desenho, T14 usou material de manipulação e produção de desenho para ampliar o raciocínio combinatório de crianças de 5 anos. Já no grupo produções imagéticas, T16 utiliza imagens produzidas pelas crianças da Educação Infantil. A última abordagem metodológica de ensino é o grupo atividades virtuais, na qual T12 usa material didático com atividades virtuais na Educação Infantil.

Os trabalhos elencados no parágrafo anterior trazem as práticas de exploração e manipulação de materiais concretos como facilitadores do processo de pensamento matemático. Como ressalta Fiorentini (1990), a prática com esses materiais pode gerar interesse e despertar situações de desafio para a criança.

\subsubsection{Quais são os instrumentos de pesquisas propostos nos trabalhos?}

Foram encontrados sete instrumentos de pesquisas propostos nos trabalhos: filmagem, fotografia, desenho, diário de campo, jogos, registros escritos e áudio. Também houve trabalhos que não 
especificaram os instrumentos de pesquisa utilizados ou, ainda, trouxeram informações confusas em relação ao assunto. Vale ressaltar, ainda, que alguns trabalhos utilizaram mais de um instrumento de pesquisa.

Quadro 8: Instrumentos de pesquisa utilizados nos trabalhos

\begin{tabular}{|c|c|c|}
\hline Instrumentos & Total de Trabalhos & Trabalhos \\
\hline Filmagem & 8 & $\mathrm{~T} 1, \mathrm{~T} 2, \mathrm{~T} 3, \mathrm{~T} 4, \mathrm{~T} 7, \mathrm{~T} 8, \mathrm{~T} 11, \mathrm{~T} 18$ \\
\hline Fotografia & 7 & $\mathrm{~T} 1, \mathrm{~T} 2, \mathrm{~T} 3, \mathrm{~T} 4, \mathrm{~T} 11, \mathrm{~T} 16, \mathrm{~T} 18$ \\
\hline Desenho & 5 & $\mathrm{~T} 1, \mathrm{~T} 2, \mathrm{~T} 3, \mathrm{~T} 12, \mathrm{~T} 13$ \\
\hline Diário de campo & 4 & $\mathrm{~T} 2, \mathrm{~T} 4, \mathrm{~T} 8, \mathrm{~T} 11$ \\
\hline Jogos & 2 & $\mathrm{~T} 5, \mathrm{~T} 15$ \\
\hline Registros escritos & 2 & $\mathrm{~T} 8, \mathrm{~T} 13$ \\
\hline Áudio & 1 & $\mathrm{~T} 11$ \\
\hline Não especificados & 4 & $\mathrm{~T} 6, \mathrm{~T} 9, \mathrm{~T} 10, \mathrm{~T} 17$ \\
\hline
\end{tabular}

Fonte: a pesquisa.

As filmagens são usadas por oito trabalhos como instrumento de produção de dados para analisar as ações e reações que as crianças demonstram durante as atividades. As fotografias são utilizadas por sete trabalhos para registrar as diversas manifestações das crianças, bem como suas experiências. Os desenhos são usados por cinco trabalhos para apresentar as noções das crianças sobre os conceitos matemáticos estudados.

Essas produções imagéticas associam-se de forma significativa com a infância. Elas permitem a captação de forma ampliada do pensamento infantil, bem como das suas estratégias de comunicação, que, muitas vezes, diferenciam-se do adulto. Lima e Nazário (2014, p. 08), por exemplo, apontam que "[...] a fotografia ajuda a registrar detalhes próprios a rituais ou a cultura material dos sujeitos, dos espaços e contexto, mas depende intrinsecamente dos conhecimentos que agregamos sobre as infâncias, bem como da disposição que temos para conhecê-las". Ou seja, o imagético permite se aproximar dessa cultura infantil, levantando possibilidades para a compreensão da sua lógica de funcionamento.

O diário de campo é utilizado por quatro trabalhos para que o pesquisador faça as suas anotações sobre o estudo e reflita sobre elas. Os jogos são usados como instrumento de pesquisa por dois trabalhos para evidenciar as interações e representações das crianças a partir da relação com o jogo. Os registros escritos aparecem em dois trabalhos para expressar a compreensão de conceitos matemáticos. Por fim, o áudio é usado por um trabalho para analisar com base na transcrição da fala das crianças os dados da pesquisa.

Tais registros emergem como uma possibilidade de memória para o pesquisador e para as crianças. O diário de campo, por exemplo, permite uma reflexão e uma revisão das práticas educacionais e de pesquisa de quem o escreve (HESS, 2009). Já os registros infantis, mesmo que não convencionais, possibilitam manifestações de falas e memórias da própria criança que o produziu. Nesse sentido, os instrumentos apresentados criam uma possibilidade de reviver e de evidenciar elementos não imediatos no campo de pesquisa. 


\subsubsection{Quais as principais referências teóricas que embasam os estudos?}

As principais referências teóricas estão divididas em duas categorias. A primeira categoria apresenta 15 trabalhos que englobam a Psicologia da Educação (Matemática). Já a segunda categoria apresenta três trabalhos que abarcam as Questões Socioculturais.

Quadro 9: Referências teóricas que embasam os estudos

\begin{tabular}{|c|c|c|}
\hline Referências Teóricas & Total de Trabalhos & Trabalhos \\
\hline $\begin{array}{c}\text { Psicologia da Educação } \\
\text { (Matemática) }\end{array}$ & 15 & $\begin{array}{c}\mathrm{T} 1, \mathrm{~T} 2, \mathrm{~T} 3, \mathrm{~T} 4, \mathrm{~T} 5 . \mathrm{T} 6, \mathrm{~T} 9, \mathrm{~T} 10, \\
\mathrm{~T} 11, \mathrm{~T} 12, \mathrm{~T} 13, \mathrm{~T} 14, \mathrm{~T} 15, \mathrm{~T} 17, \mathrm{~T} 18\end{array}$ \\
\hline Questões Socioculturais & 3 & $\mathrm{~T} 7, \mathrm{~T} 8, \mathrm{~T} 16$ \\
\hline
\end{tabular}

Fonte: a pesquisa.

Na categoria Psicologia da Educação (Matemática), encontramos trabalhos com abordagem numa perspectiva construtivista e sociointeracionaista, pautados principalmente, respectivamente, nos estudos de Piaget e Vygotsky.

Os trabalhos elaborados numa perspectiva piagetiana (T1, T3, T4, T5, T6, T10, T15) enfatizam fases do desenvolvimento e o processo de equilibração na produção do conhecimento. Além disso, tais estudos apropriam-se de autores como Duval, Vergnaud e Rabardel, que apoiam em Piaget e possuem forte impacto no campo da Didática da Matemática Francesa (PAIS, 2002).

Já os estudos com uma abordagem vigotskiana são em menor número (T2, T11, T13, T17). Estes focalizam a abordagem sociointeracionista e a perspectiva histórico-cultural de Educação Matemática, uma vez que considera que "[...] a apropriação da experiência sócio-histórica acarreta modificação da estrutura geral dos processos do comportamento e do reflexo, forma novos modos de comportamento e engendra formas e tipos de comportamento verdadeiramente novos." (LEONTIEV, [197-], p. 191).

No grupo dos trabalhos de cunho psicológico, há, ainda, aqueles que não citam de modo direto em nenhum momento Piaget e Vygotsky, mas os seus leitores em Educação Matemática (T9, T12, T14). Diferindo desse contexto, vale ressaltar T18, que propõe uma pesquisa baseada em Bruner. A intenção da pesquisa consiste em propor o debate que é possível, mesmo para a criança pequena, aprender conceitos complexos de matemática.

Na categoria Questões Socioculturais, encontramos estudos que visam abarcar a infância na sua condição mais cultural de momento de vida. T7 aproxima-se da modelagem matemática, mais especificamente dos estudos de Burak (2010), para evidenciar possíveis processos para a leitura da realidade pela criança e, consequente, apoio da matemática. Já T8 investiga a infância a partir dos referenciais da etnomatemática (D'AMBROSIO, 1990), procurando entender a criança e sua relação com o mundo cultural da matemática. Por fim, T16 elabora seu estudo a partir da concepção de infância nos estudos culturais de Kohan (KOHAN, 2004), percebendo a infância mais como um modo de viver o mundo do que uma etapa de desenvolvimento da vida.

Ao analisar as referências teóricas que embasam os estudos sobre a Educação Matemática e Educação Infantil, percebemos que o foco dos trabalhos está na psicologia, considerando mais os processos individuais de apropriação do pensamento matemático do que as questões coletivas, sociais e culturais em relação ao saber na infância. 


\section{CONSIDERAÇÕES FINAIS}

A pesquisa relatada neste artigo objetivou analisar produções científicas que discutem Educação Matemática na Educação Infantil. Para tanto, realizou-se um levantamento com os descritores "Educação Matemática" AND "Educação Infantil" no Catálogo de Teses e Dissertações da Capes (CTDC), Biblioteca Digital Brasileira de Teses e Dissertações (BDTD), Portal de Periódicos da CAPES (PPC) e no Scientific Electronic Library Online - Scielo, no período de 2010 a junho de 2020.

Os principais achados nos 18 trabalhos selecionados foram: (1) estão centrados na região Sudeste e tiveram um crescimento nos últimos cinco anos; (2) objetivam discutir o pensamento numérico, estocástico e geométrico na Educação Infantil; (3) o foco metodológico pauta-se na questão da ludicidade, principalmente sobre jogos e brincadeiras; (4) os registros imagéticos (filmagem, fotografia e desenho) são os instrumentos mais selecionados pelos pesquisadores da área; e (5) a perspectiva da psicologia tem sido a abordagem mais utilizada nos estudos, principalmente a construtivista.

Ao analisarmos os resultados, consideramos a necessidade de expansão da pesquisa em Educação Matemática na Educação Infantil, bem como a ampliação de perspectivas socioculturais nas abordagens com crianças. Nesse sentido, esperamos que as pesquisas futuras proponham, a partir deste estado de conhecimento, novas formas de pensar a área e o trabalho pedagógico.

\section{REFERÊNCIAS}

ALMEIDA, G. C. de. A classificação em crianças de pré-escola: contribuições do Flex Memo. 2017. 203f. Dissertação (Mestrado em Educação) - Universidade Federal do Ceará, Fortaleza, 2017.

ALMEIDA, I. S. de. Esquemas utilizados por crianças na educação infantil em situações envolvendo o campo conceitual de chance. 2017. 156f. Dissertação (Mestrado em Educação Matemática) - Universidade Estadual de Santa Cruz, Ilhéus, 2017.

BELO, C. B. Modelagem Matemática na Educação Infantil: Contribuições para a Formação da Criança. 2016. 150f. Dissertação (Mestrado em Ensino de Ciências Naturais e Matemática) Universidade Estadual do Centro-Oeste, Guarapuava, 2016.

BELO, P. A. de P. A aprendizagem do conceito de número de crianças do Infantil V: interações com o Flex Memo. 2018. 165f. Dissertação (Mestrado em Educação Brasileira) Universidade Federal do Ceará, Fortaleza, 2018.

BROUGÈRE, G. Lúdico e educação: novas perspectivas. Linhas críticas, Florianópolis, v. 8, n. 14, p. 5-20, 2002.

BURAK, D. Modelagem Matemática sob um olhar de educação matemática e suas implicações para a construção do conhecimento matemático em sala de aula. Modelagem na Educação

Matemática, Blumenau, v. 1, n. 1, p. 10-27, 2010.

CARVALHO, R. P. A literatura infantil e a matemática: um estudo com alunos de 5 e 6 anos de idade da educação infantil.116f. Dissertação (Mestrado em Educação Matemática) - Pontifícia Universidade Católica de São Paulo, São Paulo, 2010.

CHISTÉ, B. S. Devir - criança da matemática: experiências educativas infantis imagéticas. 2015. 106f. Tese (Doutorado em Educação Matemática) - Universidade Estadual Paulista, Rio Claro, 2015. 
D'AMBROSIO, U. Etnomatemática: arte ou técnica de conhecer e aprender. São Paulo: Editora Ática, 1990.

DIAS, C. de F. B.; PEREIRA, C. S.; DIAS, J. B.; JUNIOR, G. dos S.; APARECIDA, N.; PINHEIRO, M.; MIQUELIN, A. F. É Possível Ensinar Estocástica para Crianças da Educação Infantil? Uma Análise à Luz da Teoria de Bruner. Bolema, Rio Claro, v. 34, n. 66, jan./Apr. 2020.

DINIS, A. C. Representação semiótica: uma perspectiva para a construção do conceito de número na educação infantil. 2018. 191f. Dissertação (Mestrado em Educação, Cultura e Comunicação) - Universidade do Estado do Rio de Janeiro, Duque de Caxias, 2018.

FERNANDES, F. A.; MORAES, J. C. P.; PEREIRA, A. L. Adolescentes em conflito com a lei nas pesquisas em Educação: um olhar a partir de dissertações e teses. Revista Educar Mais, Pelotas, v. 4, n. 3, p. 773-793, 2020.

FIORENTINI, D. Uma reflexão sobre o uso de materiais concretos e jogos no Ensino da Matemática. Boletim da SBEM-SP, São Paulo, v. 4, n. 7, p. 20-35, 1990.

HESS, R. O momento do diário de pesquisa na educação. Ambiente \& Educação, Rio Grande, v. 14 , n. 1, p. $61-87,2009$.

KOHAN, W. O. (Org.). Lugares da infância: filosofia. Rio de Janeiro: D\&A, 2004.

LEONTIEV, A. N. O desenvolvimento do psiquismo humano. São Paulo: Moraes, [197-].

LIMA, P. M.; NAZÁRIO, R. Sobre a Luz do diafragma: a atribuição da fotografia na pesquisa com crianças. Educativa, Goiânia, v. 17, n. 2, p. 491-509, jul/dez, 2014.

MORAES, J. C. P. A presença da educação infantil em componentes obrigatórios de Educação Matemática em cursos de Pedagogia nas Universidades Federais do Sul do Brasil. Vidya, v. 40, n. 2, p. 5-21, 2020.

MORAES, S. P. G. de.; ARRAIS, L. F. L.; MOYA, P. T.; LAZARETTI, L. M. O ensino de matemática na educação infantil: uma proposta de trabalho com jogos. Educação Matemática Pesquisa, São Paulo, v. 19, n.1, p. 353-377, 2017.

MOREIRA, B.C.; GUSMÃO, S. R. C. T.; MOLL, V. F. Tarefas Matemáticas para o Desenvolvimento da Percepção de Espaço na Educação Infantil: potencialidades e limites. Bolema, Rio Claro, v. 32, n. 60, p. 38- 52, Jan./Apr. 2018.

OLIVEIRA, D. C. A. de. A compreensão das relações inversas da divisão por crianças nos anos iniciais de escolarização: um estudo acerca da não explicitação numérica em problemas. 2014. 89f. Dissertação (Mestrado em Psicologia Cognitiva) - Universidade Federal de Pernambuco, Recife, 2014.

PAIS, L. C. Didática da Matemática: uma análise da influência francesa. Belo Horizonte: Autêntica, 2002.

PIN, V. P. G. Jogos de reflexão pura como ferramenta lúdica para a aprendizagem matemática. 134f. Dissertação (Mestrado em Educação) - Universidade de Brasília, Brasília, 2016. 
RAMOS, A.; FARIA, P. Literacia Digital e Literacia Informacional: breve análise dos conceitos a partir de uma revisão sistemática de literatura. Linhas, Florianópolis, v. 13, n. 02, p. 102-122, jul/dez. 2012.

REZENDE, S. de. As brincadeiras e as noções espaciais na educação infantil. 2016. 173f. Dissertação (Mestrado em Educação) - Universidade Federal de Mato Grosso, Cuiabá, 2016.

RODRIGUES, N. I. Matemática, educação infantil e jogos de linguagem: um estudo etnomatemático. 2010. 83f. Dissertação. (Mestrado Profissional em Ensino de Ciências Exatas) Universidade do Vale do Taquari - Univates, Lageado, 2010.

ROMANOWSKI, J. P.; ENS, R. T. As pesquisas denominadas do tipo "estado da arte" em educação. Diálogo Educ., Curitiba, v. 6, n. 19, p. 37-50, set./dez. 2006.

SANTOS, J. S. dos. Passeios Aleatórios e o Conceito de Chance na Educação Infantil: uma análise instrumental. 2017.132 f. Dissertação (Mestrado em Educação Matemática) - Universidade Estadual de Santa Cruz, Ilhéus, 2017.

SILVA, A. de C. 0 uso de material manipulativo e a produção de desenhos no desenvolvimento do raciocínio combinatório na educação infantil. 2019. 107f. Dissertação (Mestrado em Educação Matemática e Tecnologias) - Universidade Federal de Pernambuco, 2019.

SIQUEIRA, I. G. Desenvolvimento do pensamento geométrico na educação infantil: teorias e práticas. 2019. 125f. Dissertação (Mestrado em Docência pra a Educação Básica) - Universidade Estadual Paulista, Bauru, 2019.

ZOGAIB, S. D. Sentido Espacial de Crianças na Educação Infantil: entre mapas, gestos e falas. 249f. Tese (Doutorado em Educação) - Universidade Federal do Espírito Santo, Vitória, 2019. 